\title{
REGARDING CERTAIN AERODYNAMIC SOURCES OF NON-STATIONARY FORCE LOADS ON TURBINE PROFILES
}

\author{
Tomáš Radnic*， Martin Luxa， David Šimurda \\ Institute of Thermomechanics of the Czech Academy of Sciences, Dolejškova 1402/5, Prague, Czech Republic \\ * corresponding author: radnic@it.cas.cz
}

Abstract. The paper deals with selected phenomena present in a blade cascade flow field. The presented research was performed on prismatic blade cascades composed mostly of the tip and root sections of the last stage rotor blade of a large output steam turbine. The unstarted supersonic flow on the cascade inlet, the separation of the flow and the swirl line behind the trailing edge have been identified as the possible sources of the unsteady force effects.

KeYworDs: Turbine blade cascade, unstarted supersonic flow, swirl line, off design conditions.

\section{INTRODUCTION}

The modern steam turbines of large power output utilize very long blades in the last stage of the rotor. The tip of the blade operates at supersonic inlet velocities, while the root of the blade operates at subsonic inlet velocities. In this setup, it is certain that the transonic inlet flow conditions will occur on the blade at its nominal speeds, hence unstarted supersonic flow will always be present. During the start up or shut down of the turbine, off design conditions appear in the turbine, which can generate swirl lines and flow separation. Although these phenomena can only be present during off design conditions, they can prove fatal for the turbine. Last but not least, in modern times, renewable sources are widely incorporated into the energetic grids and their non-reliable power output requires turbines to be able to operate in off design conditions on standard basis.

It is important to note the majority of phenomena leading to unsteady force effects occurs in the region of tip section which is not rigid.

\section{EXPERIMENTS}

The investigated phenomena were measured on different sections of a long turbine rotor blades made by Doosan Skoda power, formerly Škoda Pilsen. The investigation of the flow field is usually performed on several sections of the blade. Tip sections and root sections were used for creation of prismatic models that could be fitted into test section of the wind tunnel. The prismatic blades cascades with finite number of blades were assembled for maintaining best possible periodicity of the flow field that would models as best as possible the flow field in the real turbine. Perforated tailboard was utilized during measurements to prevent reflection of the shock waves and help maintaining periodicity. Blades with tailored trailing edge with sharp edges, see Figure 1 were used for investigation of the swirl line behind the trailing edge.

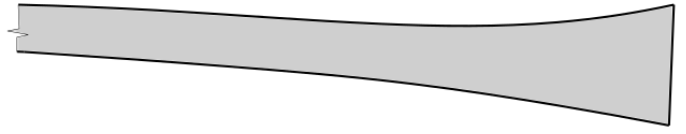

Figure 1. One of the shapes of the trailing edge used for the swirl line investigation.

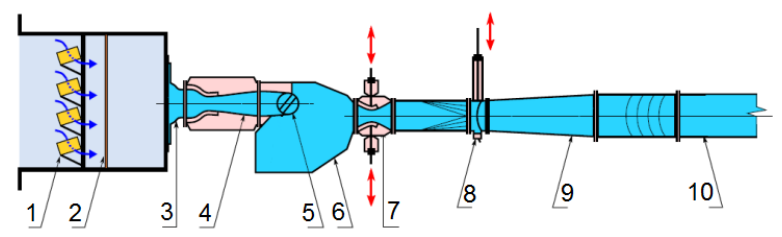

FIGURE 2. Scheme of the supersonic indraft wind tunnel. 1) silica gel drier 2) pebble filter 3) inlet nozzle 4) adjustable supersonic nozzle 5) test section 6) settling chamber 7) control nozzle 8) quick acting valve 9,10 ) duct to vacuum chamber.

Measurements were conducted in the supersonic indraft wind tunnel of the Institute of Thermomechanics of the Czech Academy of Sciences in Nový Knín. The tunnel is equipped with deformable inlet nozzle so that supersonic inlet speeds could be achieved.

\section{UNSTARTED SUPERSONIC FLOW}

The unstarted supersonic flow can be characterized as a regime, where the inner branch of inlet shock wave has a shape of a normal shock, see Figure 3 . When the inner branch of the shock wave turns into chevron shape, the flow is considered to be started supersonic flow, see Figure 4. The unstarted supersonic flow is also a boundary between transonic and supersonic flow past the cascade. In case of the unstarted supersonic flow, the inner branch of the inlet shock wave 


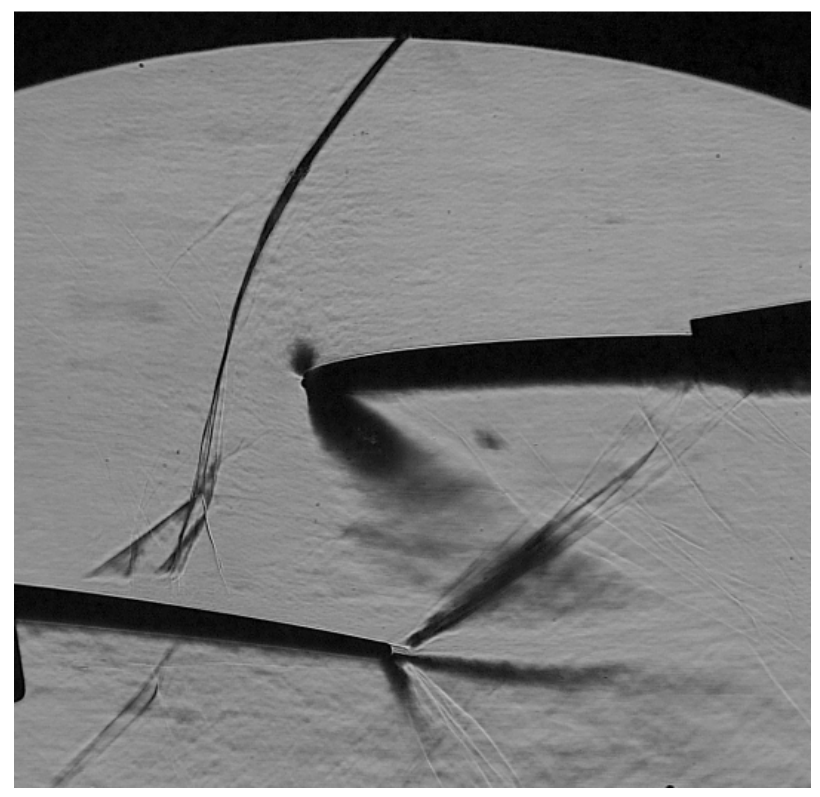

FIGURE 3. Unstarted supersonic flow field.

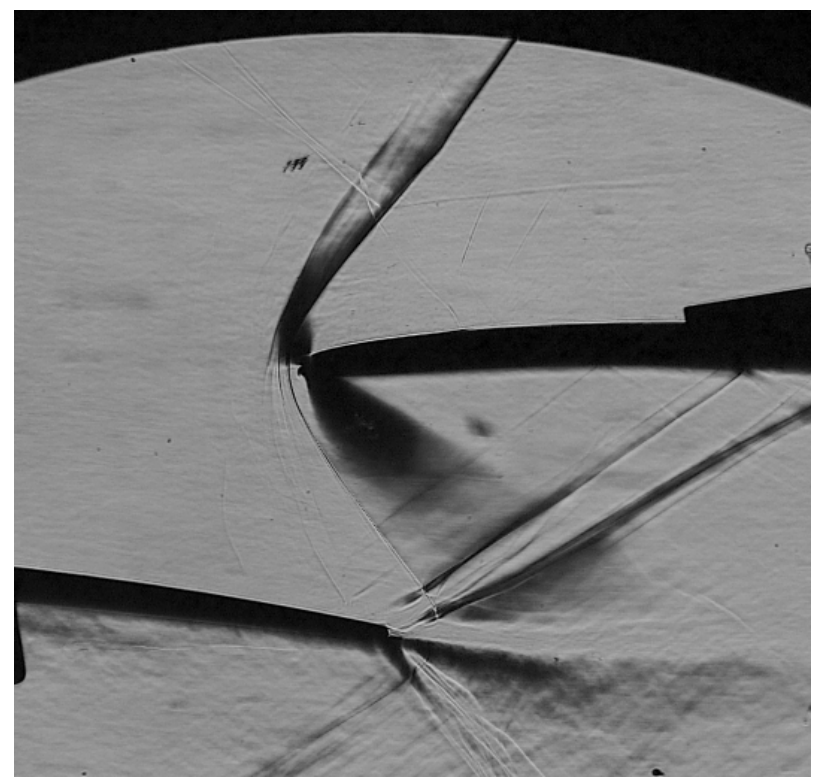

Figure 4. Started supersonic flow field.

has always unstable and unsteady behaviour and can induce instability of the whole flow field [1].

The transition of unstarted flow to started flow can be achieved by increase of inlet speed while maintaining low back pressure or by reduction of backpressure if the inlet speed is high enough.

It is important to note that the unstarted supersonic flow cannot be avoided in the turbine where the tip of the blade operates at supersonic inlet velocities. The phenomena will be present on the blade and will have force effect on the blade. The force effects need to be assessed to reckon with them during the design phase of the turbine.

The transition of unstarted supersonic flow to started supersonic flow can be seen in Figure 5 The outlet isentropic Mach number was roughly
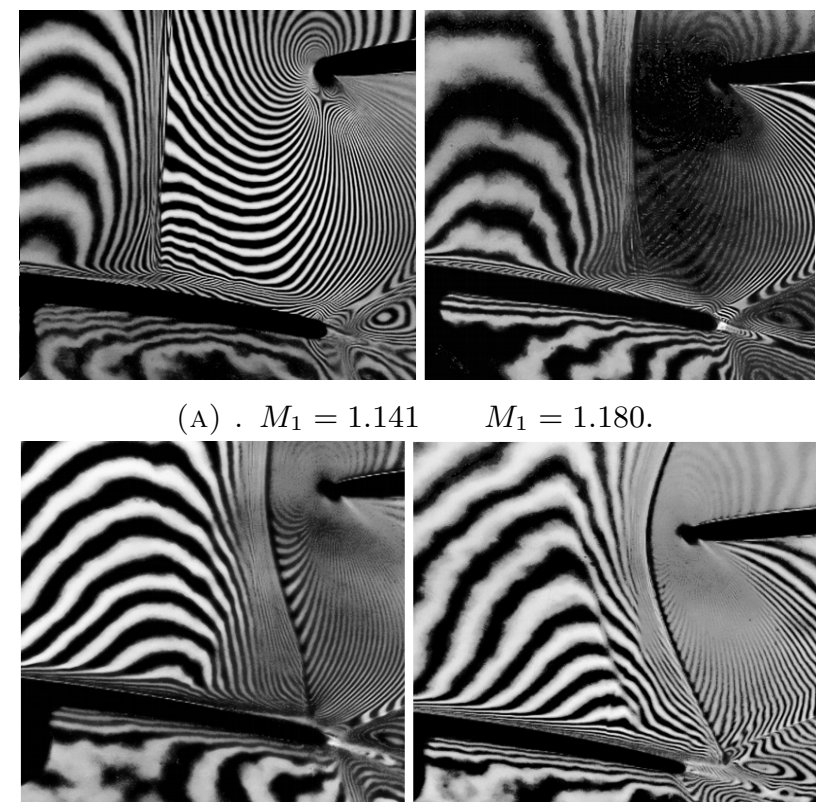

$M_{1}=1.180$.

(в) $\cdot M_{1}=1.209$

$M_{1}=1.209$.

FIGURE 5. Development of the started supersonic flow.

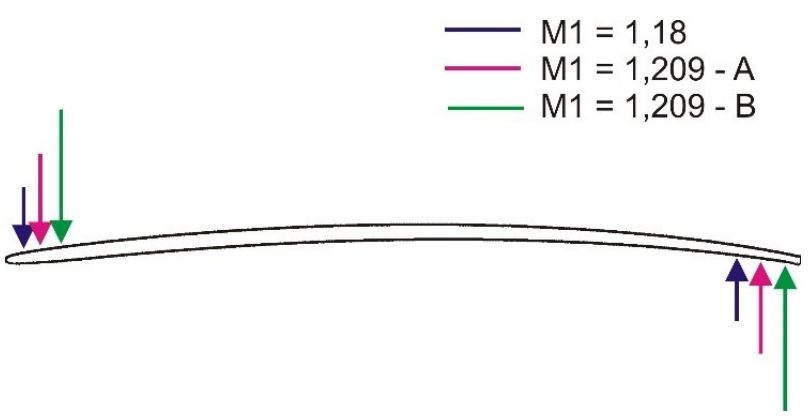

Figure 6 . Scheme of torque load of the tip of the blade with non started supersonic flow field.

$M_{2}$ is $\approx 1.8$. In the top left image, the inlet Mach number is $M_{1}=1.141$. The inlet shock wave has a shape of a normal shock wave that interacts with the boundary layer on the pressure side of the adjacent blade. The interaction causes local separation of the flow. On the top right image there is $M_{1}=1.180$. The inlet shock wave is now much closer to the leading edge and the local separation which it causes is now much more limited. On the bottom there are two images with identical inlet speed $M_{1}=1.209$. The images show unstable nature of transition between unstarted and started supersonic flow. On the left image the inner branch of the inlet shock wave hits the trailing edge of the adjacent blade, while on the right image the inlet shock wave has chevron shape. The inner branch of the inlet shock wave on the right image does not collide with the adjacent blade at all, however it can still affect the area of near wake. The shift of the shock wave shape does not have any measurable influence on the time averaged flow field parameters 2 .

The effect of this phenomenon must be investigated quantitatively to assess the induced stress in the blade. 


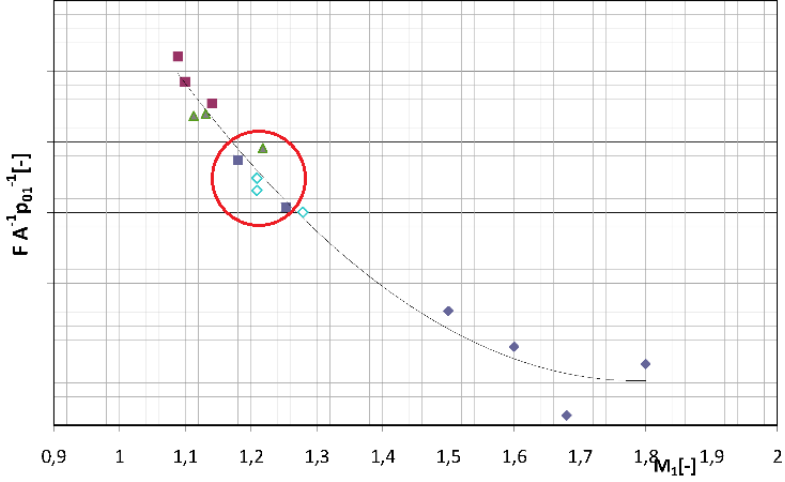

FiguRE 7. Distribution of dimensionless tangential force on the blade, points in the circle correspond to Figure 5

In the Figure 6, there is a scheme which describes force effects due to the unstarted supersonic flow. It is apparent, that the flow will induce torque stress in the blade.

Figure 7 shows distribution of the dimension-less tangential forces that affect the blade during the transition from unstarted to started supersonic flow. The torque stressing is also cyclic during the whole start up process. Because the phenomenon is unstable, even small variations in the operating conditions will result in quite large changes in the stress of the blade.

\section{FLOW SEPARATION}

The main cause of flow separation is adverse pressure gradient which induces back flow in the boundary layer. The boundary of the separation and position of the reattachment point are unsteady and are sources of pressure pulsation in the flow field. The boundary layer gains thickness and the streamlines detach from the surface at the point of zero velocity gradient. It is possible that further downstream the pressure gradient becomes more favourable and the flow reattaches to the surface. In this case the separation area is closed. Negative incidence angle can have very similar effect, the separation forms on the opposite side of the blade.

\subsection{Separation at the RoOT}

The root of the blade is much more resistant to the flow separation. The cascade has a very low pitch to chord ratio hence the density of the profiles in the cascade is high. Relatively long interblade channel and typical profile shaping provides the root section with a wide range of operational conditions in which there is no flow separation.

The experimental investigation showed, that root section cascade will maintain unseparated flow field in the range of inlet angles $\iota \in\left(-20^{\circ}, 30^{\circ}\right)$. The separation at the root does decrease the mass flow in the interblade channel by limiting the flow through area. The shift of effective shape of the profile can change the position and shape of the sonic line in the cascade.

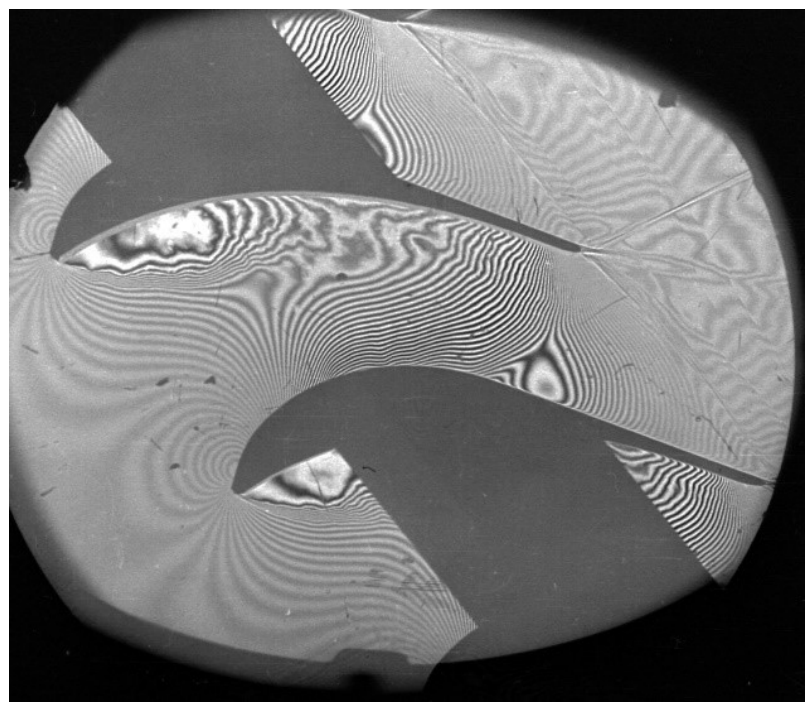

Figure 8. Flow separation on the pressure side of the blade with negative angle of incidence.
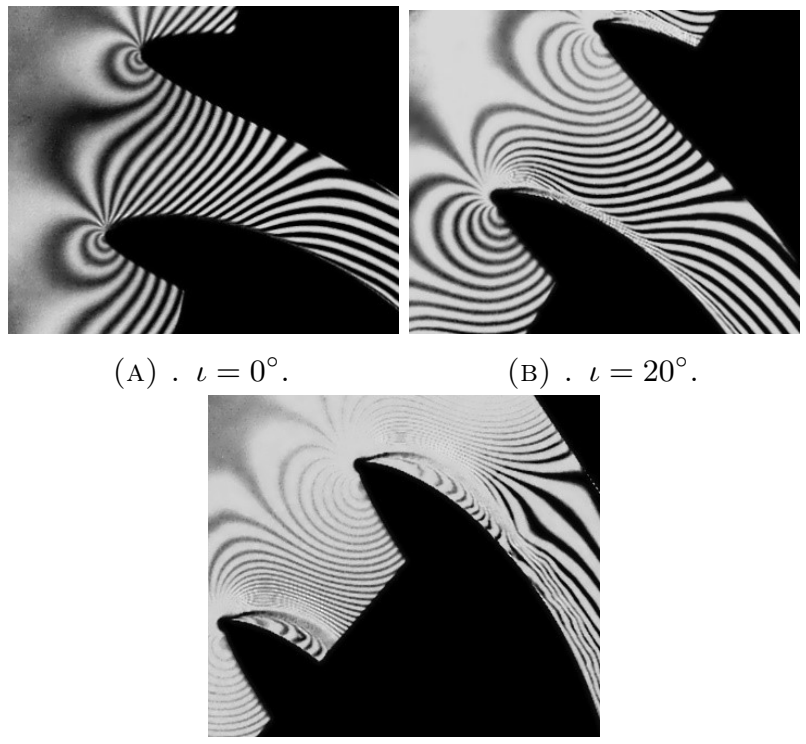

(C) $. \iota=30^{\circ}$.

Figure 9. Flow field in the root section cascade at different incidence angles, $M_{2 i s} \sim 1.5$.

\subsection{Separation in the tip Region}

The experimental investigation of the tip cascades does not allow to assess effects of varying incidence angles. The models have very high aspect ratio and the blades are thin. Excessive force effects induced by positive incidence angles will endanger the model and the measuring equipment. Negative incidence angles will lead to very low loads and increase of flow velocity in the cascade, possibly resulting in unstarted supersonic flow. The investigation can be conducted in the region of $\iota \in\left(-3^{\circ}, 3^{\circ}\right)$. Therefore, investigation of the tip section is limited, and the separation phenomena are not very well mapped.

Measurements conducted by Šafařík [3] on an older last stage blade with more robust design in the tip region had the minimal inlet angle $\iota=-6.5^{\circ}$. 


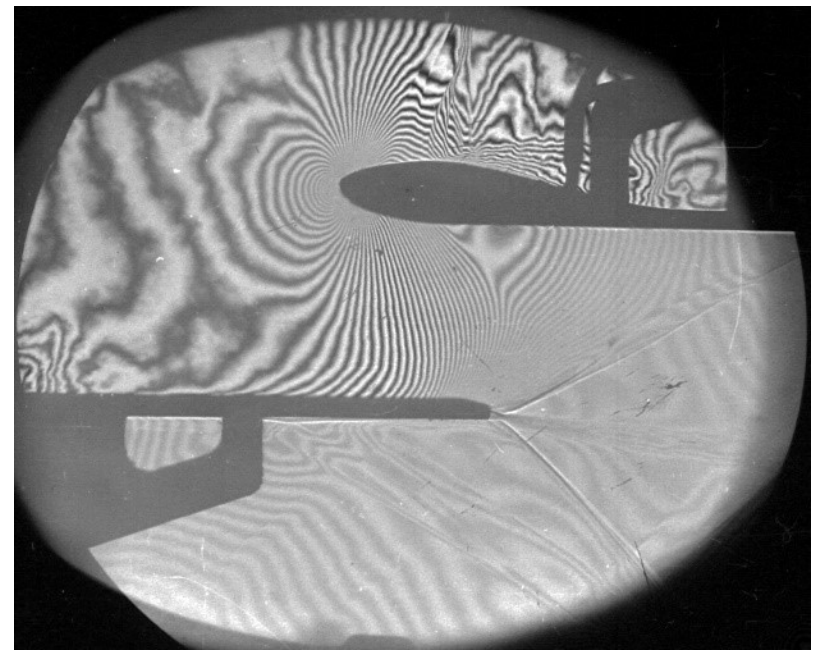

FIGURE 10. Section near the tip, $\iota=-6.5^{\circ}, M_{2 i s}=$ 1.51 .

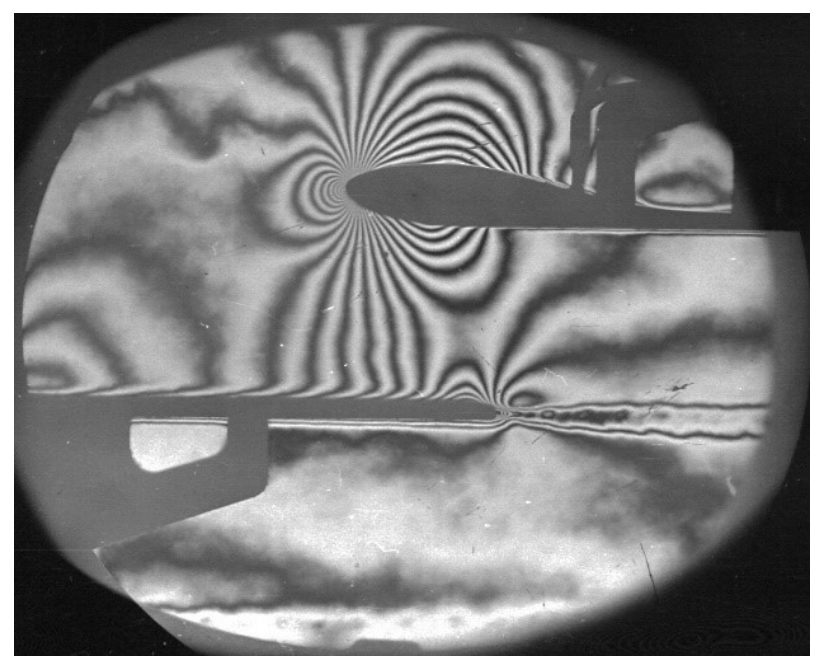

FiguRE 11. Section near the tip, $\iota=-6.5^{\circ}, M_{2 i s}=0.6$.

The measurement showed that in the area behind blade's maximal thickness there is closed area of separation, see Figure 10. This separation is caused by relatively high inlet speeds and by interaction of the inlet shock wave with the boundary layer on the adjacent blade. This separation was not present during measurements in the subsonic regions, see Figure 11.

\section{SWIRL LiNe AT THE TRAILING EDGE}

One of important aerodynamic sources of unsteady flow fields, which usually are the dominant reason of unsteady loading of blades, is a swirl line arising at the trailing edge in the near wake [4,6]. The swirl line downstream one of the blades affects the pressure distribution along a part of a suction side of the adjacent blade. This phenomenon is more noticeable in section near the tip of long blade. There, the unsteady pressure field affects (due to the geometrical arrangement of the profile cascade) a very large portion of pressure distribution on the suction side of the adjacent profile. The formation of the swirl line downstream the

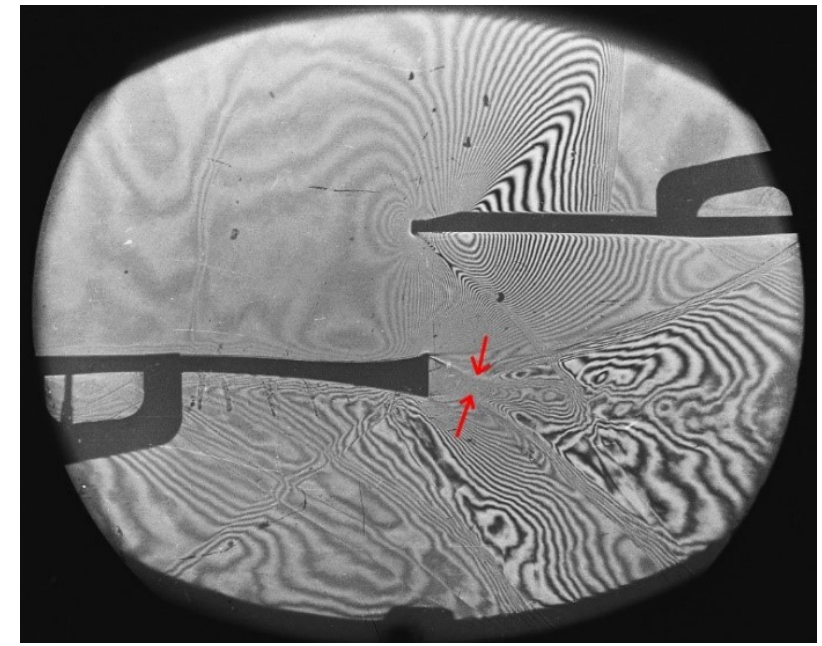

Figure 12. Tip section consisting of profiles with the tailored trailing edge, nominal condition of flow $M_{2 i s}=1.68$ [4].

trailing edge is characterized by the Strouhal number:

$$
S h=\frac{f \cdot d}{u}
$$

and by Reynolds number, for swirl line:

$$
R e=\frac{u \cdot d}{\nu}
$$

The characteristic length $d$ in case of swirl line developing at the trailing edge is the diameter of the trailing edge added to the height of the boundary layer. The criteria for evaluation of the swirl line are dependent on wide scale of flow field parameters. In a transonic flow field, the supersonic parts of the flow field in the profile cascade are subordinate to the hyperbolical description of the flow. The supersonic expansion both in the interblade channel and at the trailing edge can very favourably affect the effective dimension of the near wake, which is responsible for the swirl line origin, see Figure 12. The area of confluence at the end of the near wake, has very small characteristic length.

It is mainly caused by the large supersonic expansion on the sharp edges of the tailored trailing edge. The swirl line downstream the profile is insignificant and the pressure distribution along the suction side of the adjacent profile is stable.

The situation in the same cascade operating at off design conditions, when the flow field is significantly more subsonic, is completely different. The supersonic expansion at the trailing edge vanishes, and the decisive size for wake and swirl line formation is the real geometric dimension of the trailing edge. The very significant swirl line takes place downstream in the wake. The individual vortices float downstream the trailing edge and periodically affect the pressure distribution along the suction side of the adjacent profile, see Figure 13 . 


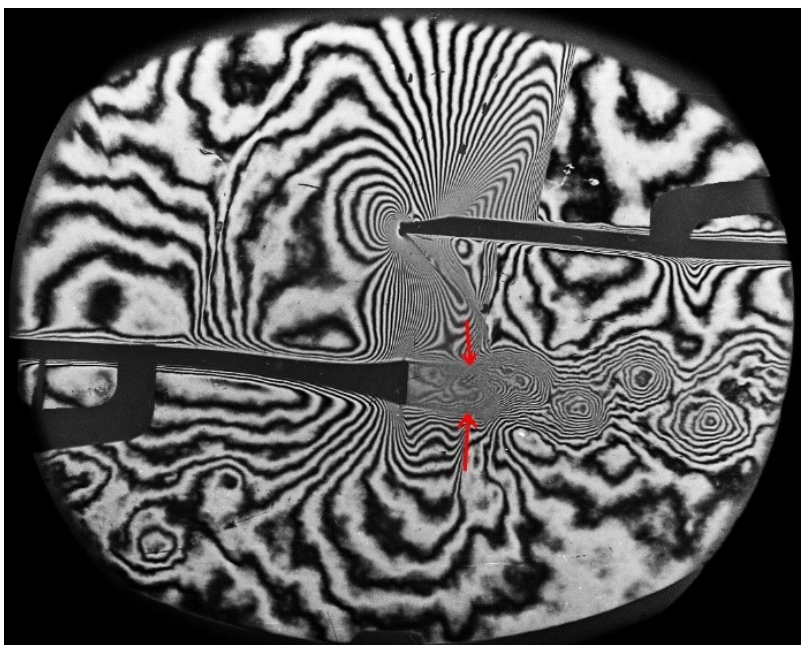

FiguRE 13. Tip section consisting of profiles with the tailored trailing edge, off-design condition of flow $M_{2 i s}=0.912$ [4].

Application of this kind of profiles in the tip region of a long rotor blade looks promising, while the aerodynamic parameters of the profile in the region of design flow conditions are good. Unfortunately, it is a problem to reach the design conditions. During the start-up process the steam turbine cannot surmount the dangerous off design regimes without seriously damaging the machine.

\section{Conclusion}

The paper deals with selected undesirable phenomena present in a blade cascade. Analysis of unstarted supersonic flow, flow separation and swirl line at the trailing edge of the blade had been conducted based on long term experimental research. The unstarted supersonic flow that is present in the design conditions proved to be unstable and transient. Force effects, especially torque, has been identified to affect the blade when unstarted supersonic flow occurs.

The flow separation does occur in non-design operating conditions, during start up, stopping or limited power output operation of the turbine. The tip region proved much more prone to detachment of the flow than the root. Incidence angle deviation $\Delta \iota=3^{\circ}$ causes separation at the tip of the blade, possibly destroying the blade cascade. The root of the blade is quite resistant to the separation which occurred at $\Delta \iota=20^{\circ}$ and $\Delta \iota=30^{\circ}$. The separation causes higher losses, sonic plane shift, pressure pulsations and other effects.

The swirl line at the trailing edge can be caused by its round shape, which cannot be avoided for technological and structural reasons. It can induce significant pressure pulsations in the flow field.
Investigation of the off design transonic flow fields in blade cascades is vital for development of turbines able to standardly operate at partial power output and that can survive frequent shutdowns and start-ups in the modern intelligent power grids.

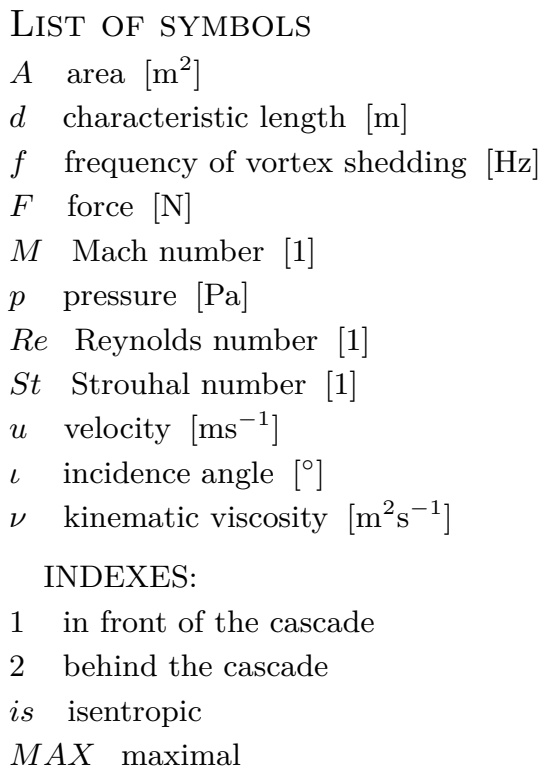

\section{ACKNOWLEDGEMENTS}

Acknowledgements of the authors belong to the Technology Agency of the Czech Republic, which supported this research under grant No. TH02020057. An institutional support RVO61388998 is also gratefully acknowledged. Special thanks are due to the DOOSAN ŠKODA POWER Co. Ltd. for making this research possible.

\section{REFERENCES}

[1] R. Dvořák, P. Šafařík, M. Luxa, D. Simurda. Optimizing the tip section profiles of a steam turbine blading. In ASME 2013 Turbine Blade Tip Technical Symposium and Course Week, Hamburg. 2013. DOI:10.1115/TBTS2013-2027

[2] M. Luxa, J. Př́hoda, D. Šimurda, et al. Investigation of the compressible flow through the tip-section turbine blade cascade with supersonic inlet. Journal of Thermal Science 25(2):138-144, 2016. DOI:10.1007/s11630-016-0844-0

[3] M. Luxa, et al. Images of flow fields in turbine profile cascades I, II, Research report IT CAS No.: Z-1382/06 (in Czech), 2006.

[4] P. Šafařík. Measurements on cascade TR-N-1, Internal report, IT CSAS (in Czech), 1981.

[5] P. Šidlof, M. Štěpán, V. Vlček, et al. Flow past a self-oscillating airfoil with two degrees of freedom: measurements and simulations. In European Physical Journal Web of Conferences, vol. 67 of European Physical Journal Web of Conferences, p. 02108. 2014. DOI:10.1051/epjconf/20146702108

[6] V. Vlček, J. Horáček, M. Luxa, J. Veselý. Visualization of unsteady flow around a vibrating profile. In 9th International Conference on Flow-Induced Vibration, Prague. 2008. 